\title{
Comparative analysis of the ribosomal DNA repeat unit (rDNA) of Perna viridis (Linnaeus, 1758) and Perna canaliculus (Gmelin, 1791)
}

\author{
Zhansheng Guo ${ }^{1}$, Leng Han ${ }^{1}$, Zhenlin Liang ${ }^{1}$, Xuguang Hou ${ }^{\text {Corresp. } 1}$ \\ ${ }^{1}$ Shandong University at Weihai, Weihai, China \\ Corresponding Author: Xuguang Hou \\ Email address: houxuguang@sdu.edu.cn
}

Perna viridis and P. canaliculus are economically and ecologically important species of shellfish. In this study, the complete ribosomal DNA unit (rDNA) sequences of these species were determined for the first time. The gene order, 18S rRNA-internal transcribed spacer (ITS) 1-5.8S rRNA-ITS2-28S rRNA-intergenic spacer (IGS), was similar to that observed in other eukaryotes. The lengths of the $P$. viridis and $P$. canaliculus rDNA sequences ranged from 8432 to $8616 \mathrm{bp}$ and from 7597 to $7610 \mathrm{bp}$, respectively, this variability was mainly attributable to the IGS region. The putative transcription termination site and initiation site were confirmed. $P$. viridis and $P$. canaliculus rDNA contained two (length: 93 and $40 \mathrm{bp}$ ) and one (length: $131 \mathrm{bp}$ ) repeat motifs, respectively. Individual intra-species differences mainly involved the copy number of repeat units. In $P$. viridis, three CpG sites with sizes of 440, 1075 and 537 bp were found to cover nearly the entire IGS sequence, whereas in P. canaliculus, two CpG islands with sizes of 361 and $484 \mathrm{bp}$ were identified. The phylogenetic trees constructed with maximum likelihood and neighbour-joining methods and based on ITS sequences were identical and included three major clusters. Species of the same genus were easily clustered together. 
1

$6{ }^{*}$ Corresponding author: Xuguang Hou;

7 Address: Shandong University at Weihai, Weihai, China

8 E-mail: houxuguang@sdu.edu.cn

Abstract: Perna viridis and P. canaliculus are economically and ecologically important species of shellfish. In this study, the complete ribosomal DNA unit (rDNA) sequences of these species were determined for the first time. The gene order, 18S rRNA-internal transcribed spacer (ITS) 1-5.8S rRNA-ITS2-28S rRNA-intergenic spacer (IGS), was similar to that observed in other eukaryotes. The lengths of the $P$. viridis and $P$. canaliculus rDNA sequences ranged from 8432 to $8616 \mathrm{bp}$ and from 7597 to $7610 \mathrm{bp}$, respectively, this variability was mainly attributable to the IGS region. The putative transcription termination site and initiation site were confirmed. $P$. viridis and $P$. canaliculus rDNA contained two (length: 93 and $40 \mathrm{bp}$ ) and one (length: $131 \mathrm{bp}$ ) repeat motifs, respectively. Individual intra-species differences mainly involved the copy number of repeat units. In $P$. viridis, three $\mathrm{CpG}$ sites with sizes of 440, 1075 and 537 bp were found to cover nearly the entire IGS sequence, whereas in P. canaliculus, two CpG islands with sizes of 361 and 484 bp were identified. The phylogenetic trees constructed with maximum likelihood and neighbour-joining methods and based on ITS sequences were identical and included three major clusters. Species of the same genus were easily clustered together. 


\section{1. Introduction}

24 The family Mytilidae comprises a diverse group of bivalves that are broadly distributed in marine environments 25 (Distel, 2000). This family includes mussels of the genus Perna, which encompasses three currently recognised 26 species of intertidal mussels: Perna viridis, P. canaliculus and P. perna. All three species are considered economically, ecologically and environmentally important (Wood et al., 2007). The green mussel $P$. viridis is a warm water bivalve species distributed along the coasts and estuaries of the Asia-Pacific region. This mollusc species is highly prized both as a food source and an important aquaculture component in southeast Asia (Tan and Ransangan, 2017; Wang et al., 2018). The green-lipped mussel P. canaliculus is endemic to New Zealand, and its range extends from the warm temperate northern waters $\left(35.5^{\circ} \mathrm{S}\right)$ to the cold temperate waters south of Stewart Island $\left(47^{\circ} \mathrm{S}\right)$ (Wood et al., 2007). The aquaculture industry surrounding the green-lipped mussel has expanded rapidly and now represents three quarters of all aquaculture exports (by value) from New Zealand (Ibarrola et al., 34 2017).

To date, molecular phylogenetic studies of Perna species have been based mainly on mitochondrial cytochrome oxidase I (COI, 623 bp in length) and ribosomal internal transcribed spacer (ITS) (ITS1 \& 2, 711 bp) sequence data (Wood et al., 2007; Cunha et al., 2014; Gardner et al., 2016). Although COI and ITS (ITS1 and ITS2) sequences can be used as molecular markers to distinguish Perna species (Wood et al., 2007), these data do not supply sufficient information for Perna classification based on geographical identification or among strains (or even individuals). Within a given locus, short amplicon sequences may include less genetic variation than longer amplicons, which would reduce the ability to distinguish closely related species (Heeger et al., 2018). Therefore, this study aimed to identify a sufficiently informative molecular marker useful for inter-species and intra-species phylogenetic analyses of Perna species.

In eukaryotes, nuclear ribosomal DNA (rDNA) is usually organised in long tandem repeats. These repeats are arranged head-to-tail in large rDNA clusters to form nucleolar organising regions (NOR) in chromosomes (Dyomin et al. 2016). Each repeated transcribed unit of rDNA comprises a coding region (18S, 5.8S and 28S rRNA genes) separated by two internal transcribed spacers (ITS1 and ITS2). Moreover, each transcribed unit is separated by an intergenic spacer (IGS) which can be subdivided into external transcribed spacers (ETSs) and the non-transcribed spacer (NTS). The coding regions are highly conserved between organisms, and have been selected as genetic markers for higher-level relationships within the molluscan (Distel, 2000; Passamaneck et al., 2004; Combosch and Giribet, 2016). Whereas non-transcribed regions (ITS1, ITS2 and IGS) exhibit high structural variability (Li et al., 2016; Huang et al., 2017; Guo et al., 2018 a). The IGS region often contains repeat fragments, promoter and enhancer sites. Both the IGS and its embedded promoters can evolve more rapidly than other parts of the ribosomal repeat unit, which can lead to considerable differences in the sequences between related species and considerable 55 intra-individual variations in length (Huang et al., 2017). The IGS region was shown to be more variable and 56 phylogenetically informative than the ITS (Zhao et al., 2011). The availability of a complete rDNA sequence would 
58 NCBI database only contains partial rDNA sequences (18S and ITS) and a complete rDNA unit has never been 59 reported.

In the present study, the complete sequences of nrDNA in $P$. viridis and $P$. canaliculus are reported for the first time, and the molecular features of various rDNA components are characterized, especially IGS. The phylogenetic relationships among members of the family Mytilidae are revealed based on ITS sequences. Comparative analyses are performed with several known complete rDNA sequences to clarify the rDNA relationships among these molluscs and other eukaryotes.

\section{2. Materials and Methods}

\subsection{Mussel material and DNA extraction}

$P$. viridis samples were collected offshore of Leizhou Bay $\left(20.968614^{\circ} \mathrm{N}, 110.325743^{\circ} \mathrm{E}\right)$ in China, and $P$. canaliculus samples were collected from Pegasus Bay $\left(43.312595^{\circ} \mathrm{S}, 172.854629^{\circ} \mathrm{E}\right)$ in New Zealand. Three individuals from each species were used in this study. Total genomic DNA was extracted from muscle tissue using the TIANamp Marine Animals DNA Kit (TianGen Biotech Co., Ltd, Beijing, China) according to the manufacturer's instructions.

\subsection{PCR amplification, cloning and sequencing}

The rDNA fragments were amplified using the primers listed in Table 1. The primers used to amplify the $18 \mathrm{~S}$ and 28S rRNA genes were designed based on partial sequences corresponding to the 18S rRNA genes of $P$. viridis (EF613234) and 28S rRNA genes of Geukensia demissa (AY145405), respectively. The primers used to amplify the 18S-ITS-28S region were designed from the $3^{\prime}$ end of P. viridis (EF613234) and 5' end of G. demissa (AY145405), while the IGS region were designed based on the $3^{\prime}$ end of G. demissa (AY145405) and 5' end of P. viridis (EF613234). Each PCR had a total volume of $25 \mu$ l and contained the following: $1 \mu \mathrm{L}$ of DNA template, $0.5 \mu \mathrm{L}$ of each primer $(10 \mu \mathrm{mol} / \mathrm{L}), 12.5 \mu \mathrm{L}$ of $2 \times$ TransTaq PCR SuperMix, and $10.5 \mu \mathrm{L}$ of $\mathrm{H}_{2} \mathrm{O}$. The reactions were initially denatured at $95^{\circ} \mathrm{C}$ for $3 \mathrm{~min}$ and subjected to 35 cycles of denaturing at $95^{\circ} \mathrm{C}$ for $45 \mathrm{~s}$; annealing at $52{ }^{\circ} \mathrm{C}$ for $18 \mathrm{~S}$ and $28 \mathrm{~S}$ rRNA, $50^{\circ} \mathrm{C}$ for $18 \mathrm{~S}-\mathrm{ITS}-28 \mathrm{~S}$ and $56.5^{\circ} \mathrm{C}$ for $28 \mathrm{~S}-\mathrm{IGS}-18 \mathrm{~S}$ for $30 \mathrm{~s}$, and extension at $72{ }^{\circ} \mathrm{C}$ for $1-3.5 \mathrm{~min}$

$82(1 \mathrm{~kb} / \mathrm{min})$. Finally, all reactions were subjected to a final extension at $72{ }^{\circ} \mathrm{C}$ for $7 \mathrm{~min}$. The PCR products were 83 electrophoresed on $0.8 \%$ agarose gels, extracted and purified. The purified PCR products were cloned using the pUCm-T Vector Cloning Kit and SanPrep Column Plasmid MiniPreps Kit (Sangon Biotech Co., Ltd.) according to the manufacturer's instructions. Three positive recombinant plasmid colonies corresponding to each amplified region were picked, cultivated and sequenced by Beijing Ruiboxingke Biotechnology Co., Ltd (Beijing, China).

\section{2.3. Sequence analysis}


90 boundaries of each region were confirmed using the NCBI database and BLAST software (Johnson et al., 2008).

91 Multiple sequence alignment was performed using T-Coffee software (Notredame et al., 2000) with manual

92 adjustments. The general molecular features of $P$. viridis and $P$. canaliculus rDNA were calculated using MEGA 7.0

93 (Kumar et al., 2016). Sub-repeat fragments and inverted repeats of the IGS region were analysed with the Tandem

94 Repeats Finder (Benson, 1999) and Unipro UGENE (Okonechnikov et al., 2012), respectively. Predictions of

95 putative transcription initiation sites (TIS) and transcription termination sites (TTSs) in the IGS regions were based

96 on a comparative analysis of the sequences with data for various marine animal species from the literature (Ki et al.,

97 2009; Luchetti et al., 2016; Chae et al., 2018; Guo et al., 2018 a). CpG islands were identified using CpGPlot

98 (Polanco and Delavega, 1994). The sequence identity was confirmed using DNAMAN software. The genetic

99 distance between $P$. viridis and P. canaliculus was calculated with MEGA 7.0 software according to a Kimura two-

100 parameter model. nucleotide diversity $(\pi)$ were calculated using the software DnaSP 5.10 (Librado and Rozas, 2009).

101 For phylogenetic reconstruction, the ITS sequences of $P$. viridis and $P$. canaliculus were first determined. Next,

102 nine complete or near-complete sequences from the following Mytilidae species were obtained from the GenBank

103 database: Mytilus edulis (AY695798), M. unguiculatus (former M. coruscus) (MK157201), M. galloprovincialis

104 (JX081670), M. trossulus (JX081669), Aulacomya maoriana (former Aulacomya atra maoriana) (DQ924557), $P$.

105 perna southern Indian population (former $P$. indica) (JQ622200), P. perna Omani population (KC692037), P. perna

106 Mauritanian population (former P. picta) (DQ924548) and Modiolus rectus (EF035114). Maximum likelihood (ML)

107 and neighbour-joining (NJ) phylogenetic trees based on ITS sequences were constructed with 1000 bootstrap

108 replicates using MEGA 7.0 software and the GTR + G nucleotide substitution and Kimura two-parameter models,

109 respectively. Crassostrea ariakensis (EU252081) was selected as the outgroup.

\section{3. Results}

\section{3.1. Complete rDNA sequences of $\boldsymbol{P}$. viridis and $\boldsymbol{P}$. canaliculus}

112

113

114

115

116

117

118

119

120

121

122

The complete rDNA units of $P$. viridis and $P$. canaliculus were determined after sequencing, comparison and assembly. Fig. 1 presents a scheme of the structural organisation of the rDNA repeat unit, which includes the following elements in order: 18S-ITS1-5.8S-ITS2-28S-IGS. The complete rDNA units of $P$. viridis and $P$. canaliculus varied from 8432 to 8616 bp and from 7597 to 7609 bp, respectively.

The length, GC content, pairwise identity and variable sites of each region are shown in Table 2. An analysis of the $P$. viridis and $P$. canaliculus sequences revealed low levels of genetic variation in the $18 \mathrm{~S}, 5.8 \mathrm{~S}$ and $28 \mathrm{~S}$ rRNA regions, which had pairwise identities of $99.56 \%, 100 \%$ and $98.94 \%$, respectively. The variable (V) sites in $18 \mathrm{~S}$ and $28 \mathrm{~S}$ rRNA made respective contributions of only $0.83 \%$ and $1.22 \%$, indicating that the coding regions $(18 \mathrm{~S}, 5.8 \mathrm{~S}$ and $28 \mathrm{~S}$ rRNA) were even more highly conserved than the complete rDNA unit (6.25\%). Differences between $P$. viridis and $P$. canaliculus were observed mainly in non-coding regions (ITS1, ITS2 and IGS). In intra-species comparisons of three individuals per species, the sequence similarities of ITS1 and ITS2 in $P$. viridis were $99.38 \%$

Peer) reviewing PDF | (2019:03:36103:3:0:NEW 5 Aug 2019) 
123 and $99.87 \%$, respectively, and these regions differed by only five and one base, respectively. Among P. canaliculus

124 individuals, both regions had sequence similarities of $100 \%$. The respective sizes of ITS1 and ITS2 were 269 and

125263 bp in P. viridis and 304 and 258 bp in P. canaliculus. Differences in the lengths and variable sites of the ITS1

126 and ITS2 regions resulted in relatively low sequence identities (66.99\% in ITS1, 67.03\% in ITS2).

127 Comparisons of the IGS sequences at various levels revealed that the highest (91.84\% for P. viridis, 98.31\%

128 for P. canaliculus) and lowest similarities (33.32\%) were found at the individual and interspecies levels,

129 respectively, and the variable sites between the IGS sequences of the two species accounted for $15.65 \%$ of all sites

130 in this region (Table 2, 3). The sizes of the IGS sequences ranged from 1399-1411 bp and 2267-2451 bp in $P$.

131 canaliculus and $P$. viridis, respectively. This result suggested that the IGS sequences of them had large differences

132 in length and a relatively high level of sequence heterogeneity. Due to heterogeneity in the length of IGSs at the

133 individual and interspecies levels of the genus Perna, the longest IGS sequence of each species was chosen as a

134 representative to outline the canonical structural organization of the IGSs from $P$. canaliculus and $P$. viridis. The

135 detailed analysis of the molecular structure of IGS sequences of them contains six distinct regions: TTS, TIS, NTS,

136 ETS, SR and CpG islands.

\section{3.2. Beginning and ending sequences of transcripts, ETS and NTS}

138

139

140

141

142

143

144

145

146

147

148

149

150

151

152

153

154

155

156

A poly (T) tract (5'-TTTTCGTTTGCCTTTTTCGTTCCTTTTTTTTT-3') was identified in the $P$. viridis IGS region from 171 to $202 \mathrm{bp}$. The sequence 5' -TTACTTGT-3' was detected in the P. canaliculus IGS region from 120 to 127 bp. Both sequences were considered putative TTSs. Accordingly, the positions of the 3' ETS sequences in the $P$. viridis and $P$. canaliculus 5 ' IGS regions were determined to correspond to bp 1-202 and bp 1127, respectively. The sequence 'TTATTATGTGGAGTGGG' was considered a putative TIS for RNA polymerase I, and the +1 position of the transcription initiation site was the $\mathrm{C} / \mathrm{T}$ nucleotide in $P$. viridis and $P$. canaliculus. The $5^{\prime}$ ETSs were localised between the putative TIS and the beginning of the 18S rRNA gene and had lengths of $581 \mathrm{bp}$ and 500-503 bp in P. viridis and P. canaliculus, respectively. NTSs comprised the remainder of IGS. The length, nucleotide diversity, intraspecies and interspecies sequence identity of $3^{\prime}$ ETS, NTS and 5' ETS in $P$. viridis and $P$. canaliculus were shown in Table 4, comparision with 3' ETS and 5' ETS, NTS had lower intraspecies and interspecies sequence identity and higher nucleotide diversity. The high level of individual, intraspecies and interspecies divergence within the NTS sequences was attributed to lower functional constraints. In contrast, the nucleotide sequences were more conserved in the ETS region than in the NTSs, despite differences in the length of the former between $P$. viridis and P. canaliculus.

\subsection{Sub-repeat (SR) regions and CpG islands in the IGS sequence}

Next, the DNA repeats in the IGS sequences were analysed to explore the IGS genetic structure in greater detail. The identification of the numbers and lengths of the repetitive sequence motifs occurring in $P$. viridis and $P$. canaliculus enabled the definition of three different SRs with lengths of 40-131bp (Fig. 1 and Fig. 2). A comparison of the IGS regions indicated an extremely high level of sequence diversity within the SR region. The P. canaliculus

Peer] reviewing PDF | (2019:03:36103:3:0:NEW 5 Aug 2019) 
157 sequence included only one type of SR (length: $131 \mathrm{bp}$ ) which was repeated 2.9 times, and three poly (A) tracts in

158

159

160

161

162

163

164

165

166

167

168

169

170

171

172

173

174

175

176

177

178

179

180

181

182

183

184

185

186

187

188

189

190

the NTS area. The $P$. viridis sequence included two repeat motifs: SR1 (length: $93 \mathrm{bp}$ ), which was repeated 9.3-11.5 times within the NTS, and SR2 (length: $40 \mathrm{bp}$ ), which was repeated 2.5 times within the 5'ETS. Differences between individuals of the same species were due to SR copy number variation. No inverted repeats were detected in either Perna species.

The GC content of the IGS region was slightly higher in P. viridis than in P. canaliculus (Table 2). Similarly, the number and lengths of the $\mathrm{CpG}$ islands were greater in $P$. viridis than in $P$. canaliculus. In $P$. viridis, three $\mathrm{CpG}$ sites were found to cover nearly the entire IGS sequence (lengths: 440, 1078 and 537 bp). By contrast, the $P$. canaliculus IGS region included two CpG islands located at the NTS and 5'ETS (lengths: 361 and 484 bp).

\subsection{Phylogenetic tree analysis based on ITS sequences}

ML and NJ phylogenetic trees were constructed based on the ITS sequences of Mytilidae species (Fig. 3). Clustering pattern analysis revealed that the NJ and ML trees were identical, both including three major clusters, and species of the same genus clearly clustered together with relatively high supporting values. For both $P$. viridis and $P$. canaliculus, the individuals were classified into single clusters with high sequence identity. All Mytilidae species included in the phylogenetic analysis belong to the subfamily Mytilinae, except M. rectus which belongs to the subfamily Modiolinae. During tree formation, M. rectus and A. maoriana initially clustered together, and then clustered with Perna and Mytilus species.

\section{Discussion}

In this study, the complete rDNA units of $P$. viridis and $P$. canaliculus were sequenced for the first time. The rDNA gene orders in these organisms were identical to those in other eukaryotes. Reported rDNA unit lengths vary among taxa, as shown in Table 5, with values of $43 \mathrm{~kb}$ (human) (Gonzalez and Sylvester, 1995) and $45 \mathrm{~kb}$ (mouse) for mammals (Grozdanov et al., 2003); $13.67 \mathrm{~kb}$ for fish (Cyprinus carpio) (Vera et al., 2003); $12.26 \mathrm{~kb}$ (Brachiola algerae) (Belkorchia et al., 2008), 9.5 kb (Plasmodiophora brassicae) (Niwa et al., 2011) and $8.3 \mathrm{~kb}$ (Eurytrema pancreaticum) (Su et al., 2018) for parasites; 7.9 kb (Paracyclopina nana) (Ki et al., 2011), 7.7 kb (Aurelia sp.1) (Ki et al., 2009) and 9.6-10.7 kb (Haliotis species) (Guo et al., 2017, $2018 \mathrm{a} \mathrm{b}$ ) for marine invertebrates; 7.9-8.9 kb (Oryza sativa) (Fujisawa et al., 2006) and 8.0-8.9 kb (Stipa spp.) (Krawczyk et al., 2017) for land plants and 11.76$12.57 \mathrm{~kb}$ (Bangia) (Xu et al., 2016) and $13.65 \mathrm{~kb}$ (Pyropia yezoensis) (Li et al., 2016) for sea algae. Meanwhile, the rDNA lengths of $P$. viridis and $P$. canaliculus were 8.6 and $7.6 \mathrm{~kb}$, respectively.

Table 5 compares each region of the rDNA unit between the two Perna species and other eukaryotes. The length of the coding region (18S, 5.8S and 28S rRNA genes) exhibits conservative features. The length of $18 \mathrm{~S}$ rRNA varies from 1799 bp to 1996 bp except for Plasmodiophora brassicae (3105 bp) and Brachiola algerae (1391 bp). The sizes of 5.8S rRNA in most eukaryotes are around $160 \mathrm{bp}$ except for Entamoeba invadens (116 bp) and Brachiola algerae. In B. algerae, the ITS size was estimated at $22 \mathrm{bp}$ and the 5.8S rRNA gene was not detected (Belkorchia et al., 2008), while the length of 28S rRNA ranged from 3 to $5 \mathrm{~kb}$. Differences in rDNA lengths among

Peer] reviewing PDF | (2019:03:36103:3:0:NEW 5 Aug 2019) 
191 species are mainly attributable to the IGS. According to the database of rDNA unit sequences submitted to GenBank,

192

193

194

195

196

197

198

199

200

201

202

203

204

205

206

207

208

209

210

211

212

213

214

215

216

217

218

219

220

221

222

223

224

225 IGS lengths vary from $0.58 \mathrm{~kb}$ in Paramphistomum cervi to over $30 \mathrm{~kb}$ in mammals.

This study observed variations in the length and sequences of the IGS region in comparisons at both intra- and inter-species levels. Length differences in this region were largely responsible for variations in the entire rDNA unit lengths of $P$. viridis and $P$. canaliculus at both levels and are mainly attributable to differences in indels between individuals. Compared with the entire IGS region and the NTS, the beginning of the 3' ETS and latter half of the 5' ETS sequences were more highly conserved and exhibited greater sequence identity ( $>65 \%)$. All rDNA IGS sequences shared characteristics such as the presence of SR elements. Therefore, heterogeneity in the IGS length can be attributed to duplications or deletions of the SR region, which may be present in different copy numbers in almost all species (Ambrose and Crease, 2011; Huang et al., 2017). In an intra-species comparison of individuals, length variants were mainly attributable to the SR zone; for example, 9.3-11.5 copies of the repeat motif SR1 were present in P. viridis individuals. Huang et al. (2017) speculated that indels are restricted to SR-rich regions. Therefore, length polymorphisms in IGS result from concerted evolution, and unequal crossover between SR elements might be a major driving force underlying the evolution of rDNA units (Ganley and Scott, 1999; Stage and Eickbush, 2007). The repeat fragments were found to be species-specific and rarely adhered to any predictable behaviour or similarities even within a genus. For example, $P$. viridis and $P$. canaliculus exhibited totally different repeat patterns, and this phenomenon has also been observed in other eukaryotes (Krawczyk et al., 2017; Guo et al., 2018 a). Possibly, the repeat pattern could be used as a genetic marker for species identification.

The IGS contains several functional elements, including TTS and TIS regions. According to Ki et al. (2009, 2011), Guo et al. (2018 a, b) and Chae et al. (2018), a poly(T) tract on the $5^{\prime}$ side of the IGS can be considered a the putative TTS in marine invertebrates. The typical structure of the region near the beginning of the 5' IGS was also observed in $P$. viridis and $P$. canaliculus, even though the sequences were not totally identical. The core promoter contains TATA and GGGG boxes, which appear to be a general feature of rDNA transcription, and this site is immediately preceded by an AT-rich region, which is commonly found in both plants and animals (Vera et al., 2003; Wang et al., 2003; Maggini et al., 2008; Krawczyk et al., 2017). However, the beginnings of the $P$. viridis and $P$. canaliculus IGS genes contained only two and one TATA-box sequences, respectively, and lacked AT-rich regions. Only one previous report described a similar putative TIS sequence (TTATTATGTGGAGTG GG). Specifically, Krawczyk et al. (2017), after an analysis of Poaceae species, also reported that an AT-rich region upstream of the TIS was not ubiquitous. The rDNA genes are widely used to resolve phylogenetic relationships between species at various taxonomic levels. However, few studies have used IGS to identify species or reveal phylogenetic relationships among mussels. A comparison of the genetic distances of rDNA between $P$. viridis and $P$. canaliculus yielded the largest value in the IGS region (0.304-0.313), which suggests that this region could be used to identify species even at a sub-genus level. Given its relatively rapid evolution (compared with other rDNA sequences) and differences in the SR sequences (e.g., copy number, sequence, and length) among species, the IGS region may be more suitable than ITS and 18/28S rRNA for reconstructing both inter- and intra-species phylogenetic relationships. 
226

227

228

229

230

231

232

233

234

235

236

237

238

239

240

241

242

243

244

245

246

247

248

249

250

251

252

253

254

255

256

257

258

259

260

261

In conclusion, we sequenced the complete rDNA unit of $P$. viridis and $P$. canaliculus for the first time. In both species, the structural organisation of the rDNA unit was similar to those of many other eukaryotes. The unit lengths in $P$. viridis and $P$. canaliculus were $8432-8616$ bp and $7597-7610 \mathrm{bp}$, respectively, and variations in the length were mainly attributable to the IGS region. We further investigated the characteristics of the IGS and assessed sequence diversity at intra-species and inter-species levels. The Perna rDNA unit provides a structural model of nuclear rDNA for molecular comparisons, particularly among Mytilidae species. These research discoveries will hopefully pave the way for analyses of mollusc population genetics and evolution.

\section{References}

Ambrose, C.D., Crease, T.J., 2011. Evolution of the nuclear ribosomal DNA intergenic spacer in four species of the Daphnia pulex complex. BMC Genet. 12, 13.

Belkorchia, A., Biderre, C., Militon, C., Polonais, V., Wincker, P., Jubin, C., Delbac, F., Peyretaillade, E., Peyret, P., 2008. In vitro propagation of the microsporidian pathogen Brachiola algerae and studies of its chromosome and ribosomal DNA organization in the context of the complete genome sequencing project. Parasitol. Int. 57, $62-71$.

Benson, G., 1999. Tandem repeats finder: a program to analyze DNA sequences. Nucleic Acids Res. 27 (2), 573-580.

Chae, J., Seo, Y., Yu, W.B., Yoon, W.D., Lee, H.E., Chang, S.J., Ki, J.S., 2018. Comprehensive analysis of the jellyfish Chrysaora pacifca (Goette, 1886) (Semaeostomeae: Pelagiidae) with description of the complete rDNA sequence. Zool. Stud. 57, 51.

Combosch, D.J., Giribet, G., 2016. Clarifying phylogenetic relationships and the evolutionary history of the bivalve order Arcida (Mollusca: Bivalvia: Pteriomorphia). Mol. Phyl ogenet. Evol. 94,298-312.

Cunha, R.L., Nicastro, K.R., Costa, J., McQuaid, C.D., Serrao, E.A., Zardi, G.I., 2014. Wider sampling reveals a non-sister relationship for geographically contiguous lineages of a marine mussel. Ecol. Evol. 4 (11), 20702081.

Distel, D.L., 2000. Phylogenetic Relationships among Mytilidae (Bivalvia): 18S rRNA data suggest convergence in mytilid body plans. Mol. Phylogenet. Evol. 15, 25-33.

Dyomin, A.G., Koshel, E.I., Kiselev, A.M., Saifitdinova, A.F., Galkina, S.A., Fukagawa, T., Kostareva, A.A., Gaginskaya, E.R., 2016. Chicken rRNA gene cluster structure. PloS ONE 11 (6), e0157464.

Fujisawa, M., Yamagata, H., Kamiya, K., Nakamura, M., Saji, S., Kanamori, H., Wu, J., Matsumoto, T., Sasaki, T., 2006. Sequence comparison of distal and proximal ribosomal DNA arrays in rice (Oryza sativa L.) chromosome 9S and analysis of their flanking regions. Theor. Appl. Genet. 113, 419-428.

Gardner, J.P.A., Patterson, J., George, S., Edward, J.K.P., 2016. Combined evidence indicates that Perna indica Kuriakose and Nair 1976 is Perna perna (Linnaeus, 1758) from the Oman region introduced into southern India more than 100 years ago. Biol. Invasions 18, 1375-1390.

Ganley, A.R., Scott, B., 1999. Extraordinary ribosomal spacer length heterogeneity in a Neotyphodium endophyte hybrid: implications for concerted evolution. Genetics. 150(4), 1625-1637. 
262 Gonzalez, I.L., Sylvester, J.E., 1995. Complete sequence of the $43 \mathrm{~kb}$ human ribosomal DNA repeat- analysis of

263

264

265

266

267

268

269

270

271

272

273

274

275

276

277

278

279

280

281

282

283

284

285

286

287

288

289

290

291

292

293

294

295

296

297

298 the intergenic spacer. Genomics 27, 320-328.

Guo, Z.S., Ding, Y., Zhang, X.H., Hou, X.G., 2017. Complete nuclear ribosomal DNA sequence analysis of Pacific abalone Haliotis discus hannai. Fish. Sci. 83, 777-784.

Guo, Z.S., Han, L., Ding, Y., Hou, X.G., Liang, Z.L., 2018 a. Molecular characterisation of the complete nuclear ribosomal DNA sequence of the blacklip abalone Haliotis rubra. New Zeal. J. Mar. Fresh. 52 (3), 430-443.

Guo, Z.S., Hou, X.G., Han, L., 2018 b. Complete nuclear ribosomal DNA sequence analyses of the black-footed abalone Haliot isiris, New Zeal. J. Mar. Fresh. 52 (2), 232-246.

Grozdanov, P., Georgiev, O., Karagyozov, L., 2003. Complete sequence of the 45 kb mouse ribosomal DNA repeat: analysis of the intergenic spacer. Genomics 82, 637-643.

Heeger, F., Bourne, E.C., Baschien, C., Yurkov, A., Bunk, B., Sproer, C., Overmann, J., Mazzoni, C.J., Monaghan, M.T., 2018. Long-read DNA metabarcoding of ribosomal RNA in the analysis of fungi from aquatic environments. Mol. Ecol. Resour. 18, 1500-1514.

Huang, Y., Yu, F., Li, X., Luo, L., Wu, J., Yang, Y., Deng, Z., Chen, R., Zhang, M., 2017. Comparative genetic analysis of the 45S rDNA intergenic spacers from three Saccharum species. PLoS ONE 12 (8), e0183447.

Ibarrola, I., Hilton, Z., Ragg, N.L.C., 2017. Physiological basis of inter-population, inter-familiar and intra-familiar differences in growth rate in the green-lipped mussel Perna canaliculus. Aquaculture 479, 544-555.

Johnson, M., Zaretskaya, I., Raytselis, Y., Merezhuk, Y., McGinnis, S., Madden, T.L., 2008. NCBI BLAST: a better web interface. Nucleic Acids Res. 36, W5-W9.

Kaukonen, J., Juselius, J.K., Tiranti, V., Kyttala, A., Zeviani, M., Comi, G.P., Keranen, S., Peltonen, L., Suomalainen, A., 2000. Role of adenine nucleotide translocator 1 in mtDNA maintenance. Science 289 (5480), $782-785$.

Ki, J.S., Kim, I.C., Lee, J.S., 2009. Comparative analysis of nuclear ribosomal DNA from the moon jelly Aurelia sp.1 (Cnidaria: Scyphozoa) with characterizations of the 18S, 28S genes, and the intergenic spacer (IGS). Hydrobiologia 616, 229-239.

Ki, J.S., Park, H.G., Lee, J.S., 2011. Extensive analysis of nuclear cistron rDNA sequence of Paracyclopina nana (Cyclopoida: Cyclopettidae). Hydrobiologia 666, 3-9.

Krawczyk, K., Nobis, M., Nowak, A., Szczecińska, M., Sawicki, J., 2017. Phylogenetic implications of nuclear rRNA IGS variation in Stipa L. (Poaceae). Sci. Rep. 7, 11506.

Kumar, S., Stecher, G., Tamura, K., 2016. MEGA7: Molecular Evolutionary Genetics Analysis version 7.0 for bigger datasets. Mol. Biol. Evol. 33, 1870-1874.

Li, X.C., Xu, J.J., He, Y., Shen, S.S., Zhu, J.Y., Shen, Z.G., 2016. The complete nuclear ribosomal DNA (nrDNA) cistron sequence of Pyropia yezoensis (Bangiales, Rhodophyta). J. Appl. Phycol. 28, 663-669.

Librado, P., Rozas, J., 2009. DnaSP v5: A software for comprehensive analysis of DNA polymorphism data. Bioinformatics 25, 1451-1452.

Luchetti, A., Scanabissi, F., Mantovani, B., 2006. Molecular characterization of ribosomal intergenic spacer in the tadpole shrimp Triops cancriformis (Crustacea, Branchiopoda, Notostraca). Genome 49, 888-893.

Peer) reviewing PDF | (2019:03:36103:3:0:NEW 5 Aug 2019) 
299

300

301

302

303

304

305

306

307

308

309

310

311

312

313

314

315

316

317

318

319

320

321

322

323

324

325

326

327

328

329

330

331

332

333

334

Maggini, F., Gelati, M.T., Spolverini, M., Frediani, M., 2008. The intergenic spacer region of the rDNA in Olea europaea L. Tree Genet. Genomes. 4, 293-298.

Niwa, R., Kawahara, A., Murakami, H., Tanaka, S., Ezawa, T., 2011. Complete structure of nuclear rDNA of the obligate plant parasite Plasmodiophora brassicae: intraspecific polymorphisms in the exon and group I intron of the large subunit rDNA. Protist 162, 423-434.

Notredame, C., Higgins, D.G., Heringa, J., 2000. T-Coffee: a novel method for multiple sequence alignments. J. Mol. Biol. 302, 205-217.

Ojha, S., Singh, N., Bhattacharya, A., Bhattacharya, S., 2013. The ribosomal RNA transcription unit of Entamoeba invadens: Accumulation of unprocessed pre-rRNA and a long non coding RNA during encystation. Mol. Biochem. Parasit. 192, 30-38.

Okonechnikov, K., Golosova, O., Fursov, M., 2012. Unipro UGENE: a unified bioinformatics toolkit. Bioinformatics $28(8), 1166-1167$.

Passamaneck, Y.J., Schander, C., Halanych, K.M., 2004. Investigation of molluscan phylogeny using large-subunit and small-subunit nuclear rRNA sequences. Mol. Phylogenet. Evol. 32, 25-38.

Polanco, C., Delavega, M.P., 1994. The structure of the rDNA intergenic spacer of Avena sativa L.: a comparative study. Plant Mol. Biol. 25 (4), 751-756.

Sone, T., Fujisawa, M., Takenaka, M., Nakagawa, S., Yamaoka, S., Sakaida, M., Nishiyama, R., Yamato, K.T., Ohmido, N., Fukui, K., Fukuzawa, H., Ohyama, K., 1999. Bryophyte 5S rDNA was inserted into 45S rDNA repeat units after the divergence from higher land plants. Plant Mol. Biol. 41, 679-685.

Stage, D.E., Eickbush, T.H., 2007. Sequence variation within the rRNA gene loci of 12 Drosophila species. Genome Res. 17(12), 1888-1897.

Su, X., Zhang, Y., Zheng, X., Wang, X.X., Li, Y., Li, Q., Wang, C.R., 2018. Characterization of the complete nuclear ribosomal DNA sequences of Eurytrema pancreaticum. J. Helminthol. 92, 484-490.

Tan, K.S., Ransangan, J., 2017. Feeding behaviour of green mussels, Perna viridis farmed in Marudu Bay, Malaysia. Aquaculture Res. 48, 1216-1231.

Vera, M.I., Molina, A., Pinto, R., Reyes, M., Alvarez, M., Krauskopf, E., Quezada, C., Torres, J., Krauskopf, M., 2003. Genomic organization of the rDNA cistron of the teleost fish Cyprinus carpio. Biol. Res. 36, $241-251$.

Wang, S.Q., Zhao, M.J., Li, T., 2003. Complete sequence of the $10.3 \mathrm{~kb}$ silkworm Attacus ricini rDNA repeat, determination of the transcriptional initiation site and functional analysis of the intergenic spacer. DNA Sequence 14 (2), 95-101.

Wang, Y.T., Zeng, Z.Y., Zhang, X.H., Shi, Q., Wang, C.G., Hu, Z.L., Li, H., 2018. Identification and characterization of a novel defensin from Asian green mussel Perna viridis. Fish Shellfish Immun. 74, 242249.

Wood, A.R., Apte, S., MacAvoy, E.S., Gardner, J.P.A., 2007. A molecular phylogeny of the marine mussel genus Perna (Bivalvia: Mytilidae) based on nuclear (ITS1\&2) and mitochondrial (COI) DNA sequences. Mol. Phylogenet. Evol. 44, 685-698.

Peer) reviewing PDF | (2019:03:36103:3:0:NEW 5 Aug 2019) 
335 Xu, J.J., Jiang, B., Chai, S.M., He, Y., Zhu, J.Y., Shen, Z.G., Shen, S.D., 2016. Complete nuclear ribosomal DNA

336

337

338

339

340

341

343 sequence amplification and molecular analyses of Bangia (Bangiales, Rhodophyta) from China. Chin. J. Oceanol. Limnol. 34 (5), 1044-1053.

Zhao, G.H., Blair, D., Li, X.Y., Li, J., Lin, R.Q., Zou, F.C., Sugiyama, H., Mo, X.H., Yuan, Z.G., Song, H.Q., Zhu, X.Q., 2011. The ribosomal intergenic spacer (IGS) region in Schistosoma japonicum: structure and comparisons with related species. Infect. Genet. Evol. 11, 610-617.

Zheng, X., Chang, Q.C., Zhang, Y., Tian, S.Q., Lou, Y., Duan, H., Guo, D.H., Wang, C.R., Zhu, X.Q., 2014.

Characterization of the complete nuclear ribosomal DNA sequences of Paramphistomum cervi. Sci. World J. 751907. 


\section{Table $\mathbf{1}$ (on next page)}

Primers used in the present paper 


\begin{tabular}{|c|c|c|c|}
\hline Amplified region & Primers & Sequences $\left(5^{\prime}-3^{\prime}\right)$ & Length $(\mathrm{bp})$ \\
\hline \multirow{2}{*}{$18 \mathrm{~S}$} & 18S-ar & CTTTCAAATGTCTGCCCTAT & \\
\hline & $18 \mathrm{~S}-\mathrm{br}$ & TTCACCTACGGAAACCTTGT & 1505 \\
\hline \multirow{3}{*}{ 18S-ITS-28S } & ITS-ar1 & AGGGACAAGTGGCGTTTAGC & 1573 \\
\hline & ITS-ar2 & TCGTAACAAGGTTTCCGTAG & 1162 \\
\hline & ITS-br & TTACCTCTAAGCGGTTTAC & \\
\hline \multirow{2}{*}{ 28S1 } & 28S1-ar & TTAGAGGTAAACGGGTGGAT & 1003 \\
\hline & 28S1-br & AGTTGATTCGGCAGGTGAG & 1093 \\
\hline \multirow{2}{*}{$28 \mathrm{~S} 2$} & 28S2-ar & GACGAAACGACCTCAACCTA & 1957 \\
\hline & 28S2-br & AATGATAGGATGAGCCGACA & \\
\hline \multirow{4}{*}{ 28S-IGS-18S } & IGS-ar1 & GGGATAACTGGCTTGTGGCA & \multirow{2}{*}{2486} \\
\hline & IGS-br1 & TGGATGTGGTAGCCGTTTCT & \\
\hline & IGS-ar2 & GGATAACTGGCTTGTGGCA & \multirow{2}{*}{3497} \\
\hline & IGS-br2 & CTGCCTTCCTTGGATGTGG & \\
\hline
\end{tabular}

1

2 


\section{Table 2 (on next page)}

Characterization of nuclear ribosomal DNA (rDNA) from Perna viridis and $P$. canaliculus bp: base pair; ITS: internal transcribed spacer; IGS: intergenic spacer; the pairwise distance and identity represent the mean value between P. viridis (F1-F3) and P. canaliculus (N1-N3), respectively. 


\begin{tabular}{|c|c|c|c|c|c|c|c|c|}
\hline \multirow{2}{*}{ Region } & \multicolumn{2}{|c|}{ P. viridis } & \multicolumn{2}{|c|}{ P. canaliculus } & \multirow{2}{*}{ Alignment length (bp) } & \multirow{2}{*}{ Pairwise identity (\%) } & \multirow{2}{*}{ Pairwise distance } & \multirow{2}{*}{ Variable sites $(\mathrm{V})$} \\
\hline & Length (bp) & GC content $(\%)$ & Length (bp) & GC content $(\%)$ & & & & \\
\hline rDNA & $8432-8616$ & $53.75-53.90$ & $7597-7610$ & $53.02-53.08$ & 8714 & 77.71 & $0.066-0.067$ & $546(6.26 \%)$ \\
\hline $18 \mathrm{~S}$ rRNA & 1799 & 50.03 & 1800 & $50.06-50.11$ & 1800 & 99.56 & $0.003-0.007$ & $15(0.83 \%)$ \\
\hline ITS1 & 269 & $52.04-53.53$ & 304 & 57.89 & 314 & 66.99 & $0.183-0.188$ & $45(14.33 \%)$ \\
\hline 5.8S rRNA & 157 & 56.05 & 157 & 56.05 & 157 & 100 & 0.000 & 0 \\
\hline ITS2 & 263 & $51.71-52.09$ & 258 & 49.22 & 277 & 67.03 & $0.246-0.252$ & $52(18.77 \%)$ \\
\hline 28S rRNA & 3678 & $54.59-54.65$ & 3679 & $54.61-54.63$ & 3679 & 98.94 & $0.009-0.010$ & $45(1.22 \%)$ \\
\hline IGS & $2267-2451$ & $55.52-55.81$ & $1399-1411$ & $52.18-52.95$ & 2486 & 33.32 & $0.304-0.313$ & $389(15.65 \%)$ \\
\hline
\end{tabular}




\section{Table 3(on next page)}

Pairwise identities (upper right matrix) and pairwise distances (lower left matrix) of IGS

F1-F3 and N1-N3 represent the three individuals of $P$. viridis and $P$. canaliculus, respectively 


\begin{tabular}{ccccccc}
\hline Sample & F1 & F2 & F3 & N1 & N2 & N3 \\
\hline F1 & & $91.48 \%$ & $98.50 \%$ & $33.32 \%$ & $33.70 \%$ & $35.75 \%$ \\
F2 & 0.013 & & $92.21 \%$ & $33.36 \%$ & $32.97 \%$ & $33.63 \%$ \\
F3 & 0.014 & 0.004 & & $35.90 \%$ & $35.39 \%$ & $36.24 \%$ \\
N1 & 0.327 & 0.323 & 0.320 & & $97.60 \%$ & $98.58 \%$ \\
N2 & 0.314 & 0.310 & 0.307 & 0.009 & & $98.30 \%$ \\
N3 & 0.318 & 0.314 & 0.311 & 0.007 & 0.007 & \\
\hline
\end{tabular}

1 


\section{Table 4 (on next page)}

The length, nucleotide diversity $(\pi)$, intraspecies and interspecies sequence identity of $3^{\prime}$ ETS, NTS and 5' ETS in P. viridis and P. canaliculus 


\begin{tabular}{ccccccccccccc}
\hline \multirow{2}{*}{ Sample } & \multicolumn{3}{c}{ Length (bp) } & \multicolumn{3}{c}{ Nucleotide diversity $(\pi)$} & \multicolumn{3}{c}{ Intraspecies identity (\%) } & \multicolumn{3}{c}{ Interspecies identity (\%) } \\
\cline { 2 - 12 } & $3^{\prime}$ ETS & NTS & $5^{\prime}$ ETS & $3^{\prime}$ ETS & NTS & $5^{\prime}$ ETS & $3^{\prime}$ ETS & NTS & $5^{\prime}$ ETS & $3^{\prime}$ ETS & NTS & $5^{\prime}$ ETS \\
\hline F1 & 202 & 1484 & 581 & & & & & & & & & \\
F2 & 199 & 1671 & 581 & 0.0067 & 0.0144 & 0.0000 & 98.51 & 88.19 & 100 & & & \\
F3 & 200 & 1485 & 581 & & & & & & & 71.20 & 54.20 & 84.23 \\
N1 & 127 & 784 & 500 & & & & & & & & \\
N2 & 127 & 769 & 503 & 0.0000 & 0.0113 & 0.0013 & 100 & 97.42 & 99.34 & & & \\
N3 & 127 & 774 & 500 & & & & & & & & & \\
\hline
\end{tabular}




\section{Table 5 (on next page)}

The rDNA unit length (nrDNA), 18S rDNA, ITS1, 5.8S rDNA, ITS2, 285 rDNA and IGS lengths (bp), and GenBank Accession numbers of eukaryots used for comparison purposes in this publication 


\begin{tabular}{|c|c|c|c|c|c|c|c|c|c|}
\hline Species & nrDNA & 18S rDNA & ITS1 & 5.8S rDNA & ITS2 & 28S rDNA & IGS & GenBank accession No. & Reference \\
\hline Homo sapiens & 42999 & 1871 & 1095 & 157 & 1155 & 5035 & 33686 & U13369 & Gonzalez \& Sylvester, 1995 \\
\hline Mus musculus & 45306 & 1870 & 1000 & 157 & 1088 & 4730 & 36462 & BK000964 & Grozdanov et al., 2003 \\
\hline Gallus gallus & 11863 & 1823 & 2530 & 157 & 733 & 4441 & 2179 & KT445934 & Dyomin et al., 2016 \\
\hline Cyprinus carpio & 13676 & 1861 & 367 & 158 & 390 & 4093 & 6807 & AF133089 and AY260899 & Vera et al., 2003 \\
\hline Entamoeba invadens & 22481 & 1962 & 116 & 116 & 78 & 3188 & 16700 & AY 190083 & Ojha et al. 2013 \\
\hline Brachiola algerae & 12269 & 1391 & & 22 & & 2557 & 7915 & AM422905 & Belkorchia et al., 2008 \\
\hline Paramphistomum cervi & $8493-10221$ & 1994 & 1293 & 157 & 286 & 4186 & $577-2305$ & KJ459934-KJ459938 & Zheng et al. 2014 \\
\hline Eurytrema pancreaticum & $8306-8310$ & 1996 & 1103 & 160 & 231 & 3669 & $1147-1151$ & KY490000- KY490004 & Su et al., 2018 \\
\hline Plasmodiophora brassicae & 9513 & 3105 & 143 & 154 & 165 & 3611 & 2332 & 1 & Niwa et al., 2011 \\
\hline Aurelia sp.1 & 7731 & 1814 & 272 & 158 & 278 & 3606 & 1603 & EU276014 & Ki et al., 2009 \\
\hline Chrysaora pacifca & 8167 & 1810 & 246 & 158 & 182 & 3609 & 2162 & KY212123 & Chae et al., 2018 \\
\hline Paracyclopina nana & 7974 & 1808 & 299 & 157 & 216 & 3572 & 1922 & FJ214952 & Ki et al., 2011 \\
\hline Haliotis discus hannai & $10668-10698$ & 1871 & 329 & 160 & 301 & 3411 & $4624-4654$ & KY485141- KY485146, KY569413-KY569414 & Guo et al., 2017 \\
\hline Haliotis iris & 9579-9706 & 1858 & 321 & 160 & 296 & 3412 & $3560-3662$ & KY933301-KY933305, KY978225-KY978226 & Guo et al., 2018 \\
\hline Haliotis rubra & 9881 & 1858 & 327 & 160 & 297 & 3413 & 3854 & $\begin{array}{l}\text { MF099780, MF111108, MF106175, } \\
\text { MF106174, MF099782, MF113042 }\end{array}$ & Guo et al., 2018 \\
\hline Oryza sativa & $7928-8934$ & / & / & l & / & 1 & l & OSJNOa063K24 and OSJNBb0013K10 & Fujisawa et al., 2006 \\
\hline Stipa spp. & $7791-8897$ & 1811 & 221 & 164 & $205-207$ & 2193-3098 & 3397 & KY826229- KY826235 & Krawczyk et al., 2017 \\
\hline Bangia & $10976-11497$ & 1839 & $217-699$ & 158 & $398-437$ & 3751 & 4613 & KР279672-KP279682, KP311305-KP311315 & Xu et al., 2016 \\
\hline Pyropia yezoensis & $13650-13654$ & 1834 & $371-372$ & 159 & $532-535$ & 4770 & 5984 & KJ578745-KJ578748, KJ608639-KJ608640 & Li et al., 2016 \\
\hline Marchantia polymorpha & 16103 & 1820 & 1067 & 158 & 381 & 3468 & 9209 & $\mathrm{AB} 021684$ & Sone et al., 1999 \\
\hline P. viridis & $8432-8616$ & 1799 & 269 & 157 & 263 & 3678 & $2267-2451$ & MK419104-MK419106 & Present study \\
\hline P. canaliculus & $7597-7610$ & 1800 & 304 & 157 & 258 & 3679 & $1399-1411$ & MK419107-MK419109 & Present study \\
\hline
\end{tabular}




\section{Figure 1}

Structural organisation scheme of nuclear ribosomal DNA (nrDNA) repeat units in $P$. viridis and $P$. canaliculus.

ITS: internal transcribed spacer; IGS: intergenic spacer; ETS: external transcribed spacer; NTS: non-transcribed spacer; TTS: transcription termination site; TIS: transcription initiation site; SR: sub-repeat; $\mathrm{CpG}$ island: cytosine-guanine island. The arrows represent primer positions.
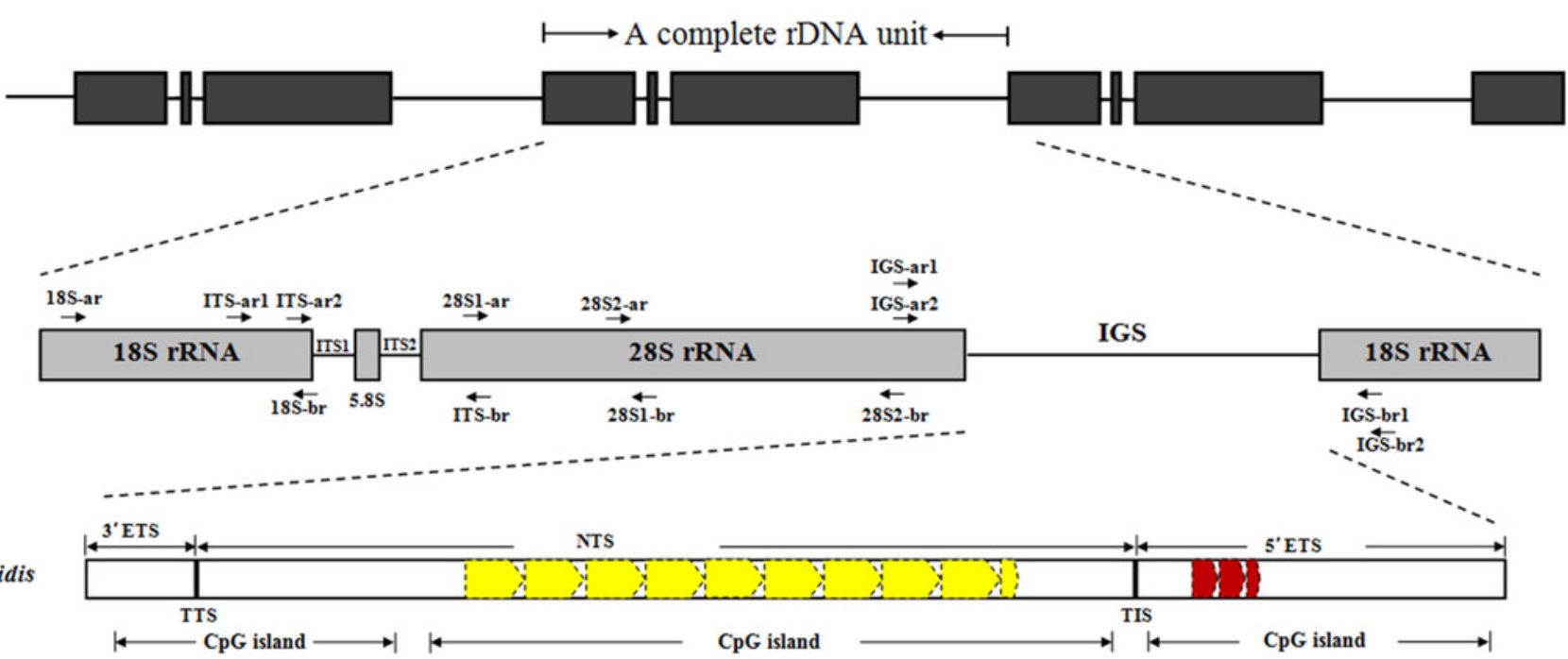

P. canaluculus

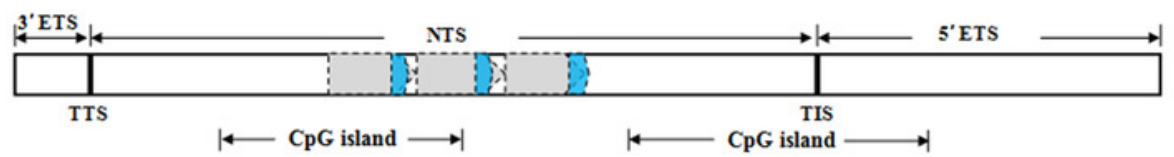


Figure 2

Alignment of the sub-repeat region of $P$. viridis and $P$. canaliculus.

SR1 (Length: $93 \mathrm{bp}$ ) and SR2 (Length: $40 \mathrm{bp}$ ) belong to $P$. viridis, and SR3 (Length: $131 \mathrm{bp}$ ) belong to $P$. canaliculus. Two nucleotide bases are identical and marked in gray. Three nucleotide bases are identical and marked in black.

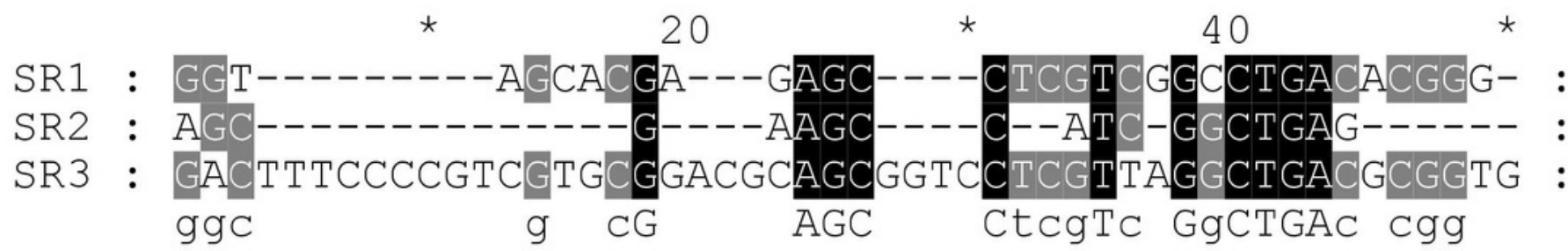

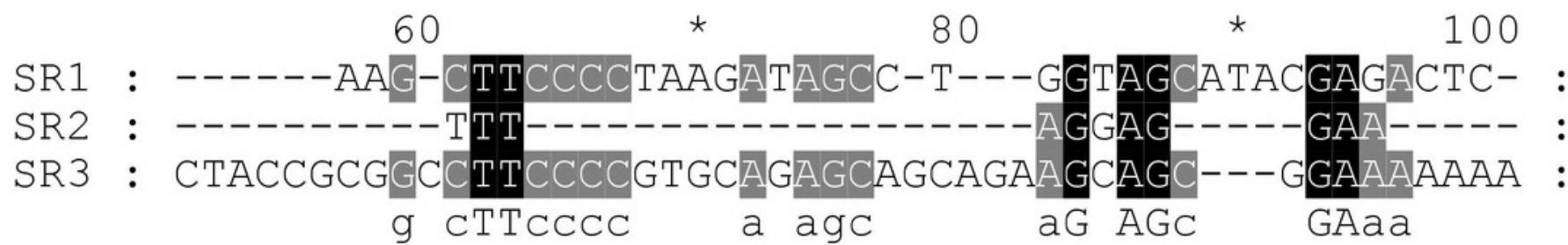

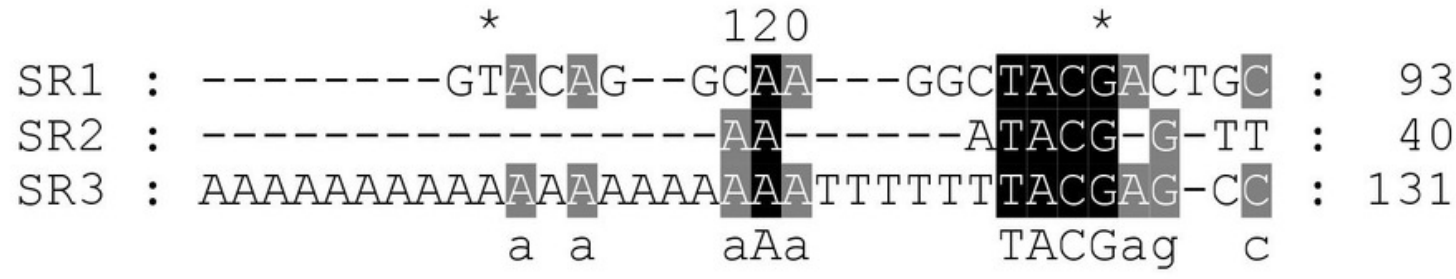


Figure 3

Phylogenetic trees constructed using maximum likelihood (ML) and neighbour-joining (NJ) methods based on ITS sequences of Mytilidae species.

Numbers around the branches indicate bootstrap support from 1000 tests. ML and NJ trees are topologically identical. F1-F3 and N1-N3 represent the three individuals of $P$. viridis and P. canaliculus, respectively.

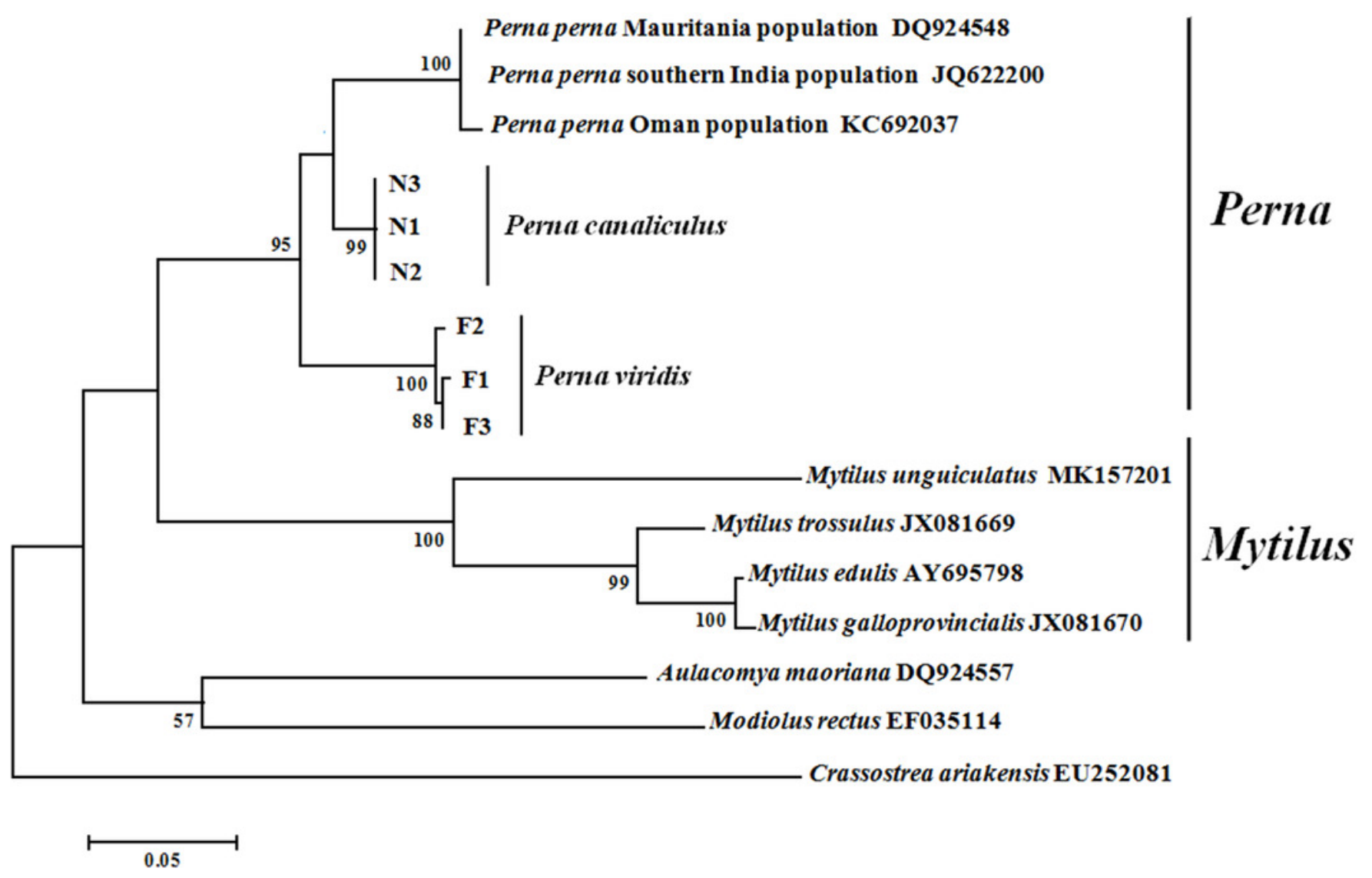

